\title{
Screening of Malaysian indigenous microalgae for antioxidant properties and nutritional value
}

\begin{abstract}
Fourteen indigenous microalgal samples from Malaysia were isolated, purified and cultured from fresh,brackish and marine waters. The ability of the microalgae to be natural sources of antioxidants was studied by a screening test using three antioxidant chemical assays[ferric thiocyanate (FTC), thiobarbituric acid (TBA) and 1, 1'-diphenyl-2-picrylhydrazyl (DPPH)]. The results showed that six microalgal methanolic crude extracts(Isochrysis galbana, Chaetoceros calcitrans, Scenedesmus quadricauda, Chlorella vulgaris, Nannochloropsis oculata and Tetraselmis tetrathele) were active in inhibiting the lipid peroxidation of linoleic acid. Among all the microalgae, I. galbana and C. calcitrans showed the highest antioxidant activity $(>90 \%)$ in FTC and TBA assays, indicating that these microalgae might contain active compounds for protection from lipid peroxidation. Nutritional analyses were performed on microalgae with high antioxidant activities (I. galbana and C. calcitrans) in order to investigate their nutritive value. Both microalgae were found to be rich in nutrients. For examples, I. galbana had average percentage composition of protein, carbohydrate, and lipid, as $47.9 \pm 2.5 ; 26.8 \pm 0.2 ; 14.5 \pm 1.4 \%$, respectively,while the corresponding values for $\mathrm{C}$. calcitrans were $36.4 \pm 1.7 ; 27.4 \pm 3.0 ; 15.5 \pm 0.9 \%$. In addition, they contained high levels of omega-3 polyunsatrated fatty acids (PUFA) $(28.0 \% \pm 0.7$ in I. galbana and $28.5 \% \pm 1.4$ in C. calcitrans),omega-6 PUFA (6.5\% \pm 1.8 in I. galbana and $23.0 \% \pm 2.5$ in C. calcitrans) and a high composition of essential amino acids. This study illustrates that some microalgae such as I. galbana and C. calcitrans have the potential to be used as natural sources of antioxidants with high nutritional value.
\end{abstract}

Keyword: Amino acids, Antioxidant activity, Fatty acids, Microalgae 